\title{
EXTRINSIC CPT VIOLATION WITH STERILE NEUTRINO
}

\author{
Sujata Diwakar ${ }^{1}$, Jyotsna Singh ${ }^{2}$, Yogita Pant ${ }^{3}$, R.B.Singh ${ }^{4}$ \\ ${ }^{1,2,3,4}$ Department of Physics, University of Lucknow, Lucknow-226007, India \\ * Corresponding Author: jyo2210@yahoo.co.in
}

\begin{abstract}
In this work, the matter induced CPT violating effects in neutrino oscillations are quantified with the help of Neutrino Factory setup. Here this extrinsic CPT violation is checked for the disappearance channel $\left(v_{\mu} \rightarrow v_{\mu}\right)$. The information of extrinsic CPT violation will help us in investigating pure CPT violation. The profile of interior density of earth from which the neutrinos passes is considered as step function matter density profile (PREM) in the present work. In the considered $(3+1)$ neutrino framework, extrinsic CPT violation is checked for baselines $1300 \mathrm{~km}-7500 \mathrm{~km}$ for energy range 4 to $50 \mathrm{GeV}$. In order to check sterile contribution in the total extrinsic CPT violation, we have selected few observables which can disentangle the contributions arising due to the presence of sterile neutrino from those which arises due to the presence of three active neutrinos. In the presence of sterile neutrino these observables promote higher energies (more than $20 \mathrm{GeV}$ ) especially $15 \mathrm{GeV}$ for long baseline experiments to probe pure(intrinsic) CPT violation because sterile contribution to extrinsic CPT violation becomes very less at these energies. To give a realistic estimate of extrinsic CPT violation, the product of sterile angles $\theta_{24}$ and $\theta_{34}$ is constrained in our analysis. We get $\theta_{24} \cdot \theta_{34} \gtrsim 0.006 \mathrm{rad}^{2}$ with liquid argon detector and $\theta_{24} \cdot \theta_{34} \gtrsim 0.007 \mathrm{rad}^{2}$ with magnetized iron detector at $90 \%$ C.L. for the observance of extrinsic CPT violation.
\end{abstract}

Keywords: CPT Violation, Sterile Neutrino, Neutrino Factory, Extrinsic CPT violation

\section{INTRODUCTION}

Confirmation of neutrino oscillations by different neutrino experiments like SNO (Sudbury Neutrino Observatory) [1] [2], Kamiokande and Super- Kamiokande [3] assigned mass to the neutrinos. Whereas in standard model (SM) neutrinos are massless. In an attempt to accommodate these tiny masses of neutrinos in the standard model, a simple extension in this model was required. This simple extension in the standard model was able to accommodate two mass square differences observed from neutrino oscillation experiments. Whereas the final results announced by the LSND collaboration [4] [5] [6] [7] [8] [9] for $\bar{v}_{\mu} \rightarrow \bar{v}_{e}$ appearance channel gave evidence of neutrino oscillations with large frequency, indicating the presence of atleast one neutrino with mass greater than $0.4 \mathrm{eV} / \mathrm{c}^{2}$. Even the simplest extensions of the standard model were not able to explain this anomaly. This inconsistency in neutrino mass spectrum was explained by introducing sterile neutrinos along with three active neutrino of SM. The number of active neutrinos are restricted to be three by the LEP data [10] [11][12] from the measurement of invisible $\mathrm{Z}_{0}$ boson decay width. Hence to explain LSND data which was further supported by MiniBooNE experiment, sterile neutrino was introduced. As the mass square differences of three active neutrinos were very small in comparison to the observed one, this sterile neutrino was thought to be responsible for large frequency oscillations. The number of sterile neutrinos can vary from one to any integer N. LSND anomaly or the sterile neutrino concept implies that there may be violation of CPT invariance. CPT symmetry is a fundamental symmetry of quantum field theory. This symmetry comprises of three individual, C,P and T symmetries of the standard model. CPT symmetry indicates that all physical laws must remain invariant under the combined operation of above three i.e charge conjugation $(\mathrm{C})$,parity inversion $(\mathrm{P})$ and time reversal( $\mathrm{T})$. As we know neutrino masses gave first dent in the SM, this particle is extensively used to probe physics beyond the standard model. Several studies, using neutrinos as a probe for observing CPT violation signals are regularly being carried out[13] [14] [15][16] [17].

Our work is organized in four steps. In the beginning we have discussed $(3+1)$ neutrino probability equations and at the second step we talk about the simulation technique used for our analysis. Third step deals with the results produced out of simulated environment for the observance of CPT violation. And at last we have discussed our results.

\section{NEUTRINO TRANSITION PROBABILITY}

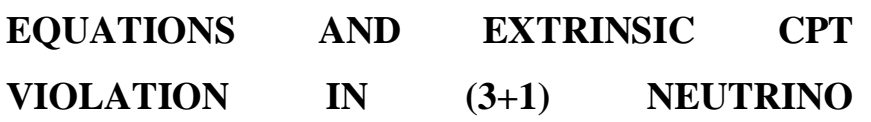

\section{FRAMEWORK}

A general expression for the three flavor conversion probabilities in neutrino oscillations can be written as

$$
\begin{aligned}
P_{\alpha \beta}= & \delta_{i j}-4 \sum_{i>j} \operatorname{Re}\left[\tilde{U}_{\alpha i} \tilde{U}_{\alpha j}^{*} \tilde{U}_{\beta i}^{*} \tilde{U}_{\beta j}\right] \sin ^{2} \Delta_{i j} \\
& +2 \sum_{i>j} \operatorname{Im}\left[\tilde{U}_{\alpha i} \tilde{U}_{\alpha j}^{*} \tilde{U}_{\beta i}^{*} \tilde{U}_{\beta j}\right] \sin 2 \Delta_{i j}
\end{aligned}
$$

In the above equation $P_{\alpha \beta}$ denotes the transition probability of neutrino from $v_{\alpha}$ to $v_{\beta}$ flavor where $\alpha, \beta$ can be e, $\mu, \tau$ and $\mathrm{i}, \mathrm{j}=v_{1}, v_{2}, v_{3}$. Neutrino transition probabilities obtained using perturbative expansions in appropriate small dimensionless 
parameters with $\Delta_{31}=\mathrm{O}(1)<<\Delta_{41}$ are used in this work [19]. For small angles $\left(\theta_{i j} \simeq \sin \theta_{i j} \simeq s_{i j}\right)$ neutrino oscillation probabilities are found as.

$$
\begin{aligned}
P_{e e}=1 & -2 s_{14}^{2}-4 s_{13}^{2} \Delta_{31}^{2} \frac{\sin ^{2}\left(\Delta_{31}-\Delta_{e}\right)}{\left(\Delta_{31}-\Delta_{e}\right)^{2}} \\
P_{\mu \mu}= & 1-2 s_{24}^{2} \cos ^{2} \Delta_{31}-\left(1-8 s_{23}^{2}\right) \sin ^{2} \Delta_{31} \\
& +\left(c_{12}^{2} \Delta_{12}-2 s_{24} s_{34} \cos \delta_{3} \Delta_{n}\right) \sin 2 \Delta_{31} \\
& +\frac{s_{13}^{2} \Delta_{31}}{\left(\Delta_{31}-\Delta_{e}\right)^{2}}\left[2 \Delta_{31} \sin \Delta_{e} \cos \Delta_{31}\right. \\
& \times \sin \left(\Delta_{31}-\Delta_{e}\right)-\left(\Delta_{31}-\Delta_{e}\right) \Delta_{e} \\
& \left.\times \sin 2 \Delta_{31}\right]
\end{aligned}
$$

The above equations can be analysed as ;

- In probability equation $P_{e e}, \theta_{14}$ appears in the constant leading order term. The $v_{e} \rightarrow v_{e}$ disappearance channel can be used to constrain sterile mixing angle $\theta_{14}$.In this equation $\Delta_{e}$ and $\Delta_{n}$ (matter potential terms) terms are not present along with the sterile parameter terms hence values of sterile parameters will not effect the extrinsic CPT violation.

- Terms $\theta_{24}, \theta_{34}$ and $\delta_{3}$ are present in the probability equation $P_{\mu \mu}$ after the first order. The constraints on these values will effect the disappearance probability $P_{\mu \mu}$. The $\Delta_{e}$ and $\Delta_{n}$ (matter potential terms) terms are present in the leading order terms of the probability equation. These terms will give rise to extrinsic CPT violation. By varying energy and baseline we can check the potential of neutrino factory setup to capture extrinsic CPT violation.

Hence for this work, we select $v_{\mu} \rightarrow v_{\mu}$ channel to probe extrinsic CPT violation .

The CPT symmetry indicates

$$
P_{\nu_{\alpha} \longrightarrow \nu_{\beta}}=P_{\bar{\nu}_{\beta} \longrightarrow \bar{\nu}_{\alpha}}
$$

$$
\Delta P^{C P T}=P_{\left(\nu_{\alpha} \rightarrow \nu_{\beta}\right)}-P_{\left(\bar{\nu}_{\beta}-\bar{\nu}_{\alpha}\right)}=0
$$

Here bar symbol is used for the antineutrinos. If CPT is conserved $\triangle P^{C P T}$ should be zero. Due to the presence of matter in the path of neutrinos and antineutrinos we get some extrinsic CPT violation which makes $\triangle P^{C P T} \neq 0$ (assuming that the CPT invariance theorem holds), this nonzero value of probability difference is known as fake CPT or extrinsic CPT violation. By observing $\triangle P^{C P T}$ we can quantify the extrinsic CPT violation in presence of sterile neutrino.

In an attempt to quantify the extrinsic CPT violation arising due to the presence of sterile neutrino, at probability level we observe the CPT probability difference $\triangle P_{\alpha \beta}^{C P T}$ given by

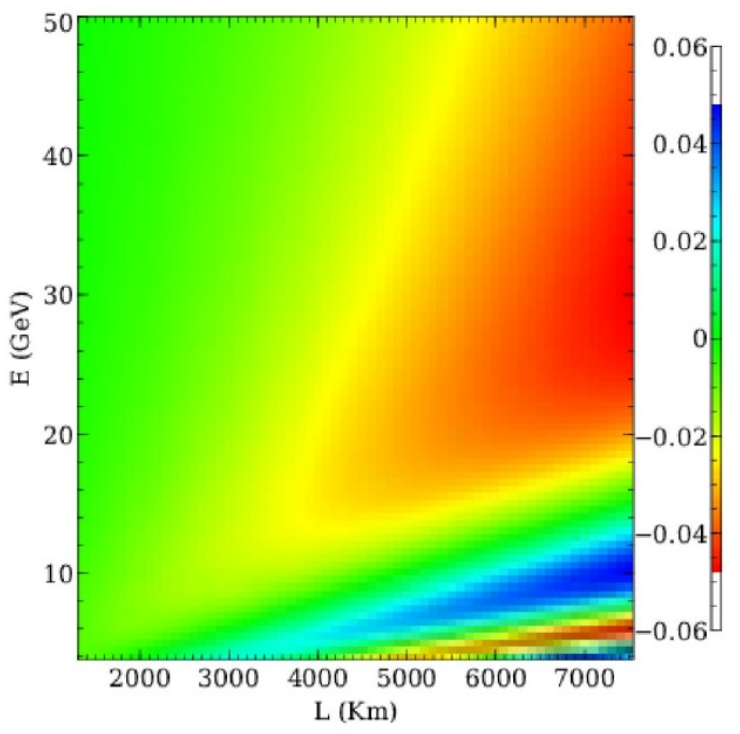

Fig 1: Normal hierarchy oscillograph demonstrates variations of $\triangle P_{\mu \mu}^{C P T}$ with baseline and energy.

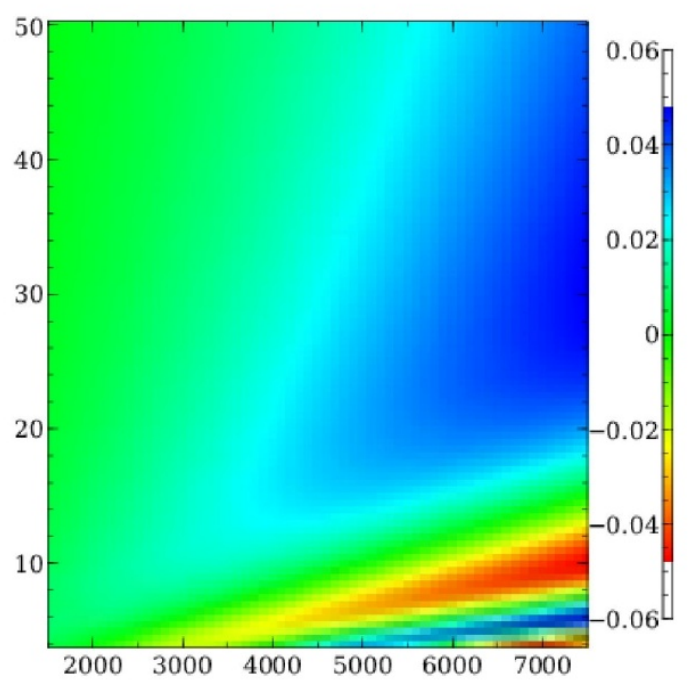

Fig. 2: Inverted hierarchy oscillograph demonstrates variations of $\triangle P_{\mu \mu}^{C P T}$ with baseline andenergy.

$$
\triangle P_{\alpha \beta}^{C P T}=\left(P_{\alpha \beta}-P_{\bar{\beta} \bar{\alpha}}\right)_{4 \nu}-\left(P_{\alpha \beta}-P_{\bar{\beta} \bar{\alpha}}\right)_{3 \nu}
$$

The probability equation (3) is used to check $\Delta P_{\alpha \beta}^{C P T}$ for $v_{\mu} \rightarrow v_{\mu}$ channel with neutrinos, and for antineutrinos we replace $\Delta_{e / n}$ by $-\Delta_{e / n}$ and $\delta_{j}$ by $-\delta_{j}$ in the same equation. Substituting the oscillation probabilities of neutrinos and antineutrinos in equation (6), we get 


$$
\triangle P_{\mu \mu}^{C P T} \approx 4 s_{24} s_{34} \Delta_{n} \cos \delta_{3} \sin 2 \Delta_{31}
$$

For $v_{\mu} \rightarrow v_{\mu}$ channel extrinsic CPT violation depends on the sterile mixing angles $\theta_{24}$ and $\theta_{34}$, the matter potential $\Delta_{n}$ originating from neutral current interactions $A_{n}$ and dirac phase $\delta_{3}$.

\section{SIMULATION TECHNIQUES}

Neutrino factory [20][21][22][23][24][25] will be a suitable choice for probing CPT violation, as it will provide higher signal rates with lower background rates in comparison to conventional beam. A $50 \mathrm{GeV}$ neutrino factory with $1.4 \times$ $10^{21}$ useful muon per polarity per year is taken into consideration for the entire analysis. In order to reduce systematical errors, near detector is also used along with the far detector. The systematical errors considered for this analysis are given in table [III]. A very good detector with high energy and angular resolution is required for meaningful observation. This view point lead us to select two different detectors

(i) Liquid Argon detector as Near and Far Detector (Energy Resolution $\frac{\Delta E}{G e V}=20 \sqrt{E / G e V}$ for $v_{\mu}$ ) in neutrino factory setup.

(ii) Magnetized Iron detector as Near and Far Detector (15\% energy resolution for muon) in neutrino factory setup.

A near detector of mass 200 tons is placed at a distance 20 $\mathrm{m}(\mathrm{L})$ from the end of the decay straight of the muon storage ring. In case of near detector we have substituted the effective baseline $(48.98 \mathrm{~m})$ in place of baseline $(\mathrm{L})$ for our calculations. This effective baseline is calculated using eq. $L_{e f f}=\sqrt{d(d+s)}$ as given in ref. [26]. The far detector of fiducial mass $50 \mathrm{Kt}$ is kept at a distance $7500 \mathrm{Km}$.

The general long baseline experiment simulator GLoBES [27] [28] is used to simulate the extent of extrinsic CPT violation that can be captured with neutrino factory. In present work we input the four flavor neutrino conversion probability in the user defined probability engine of GLoBES.

An uncertainty of 5\% on matter density is imposed [29][30]. The best fit values of standard oscillation parameters in three neutrino framework[18] are mentioned in the table[I], whereas for $\Delta m_{41}^{2}=0.1 \mathrm{eV}^{2}$ the values of sterile parameters[19] are mentioned in table[II]. These best fit values are used in our analysis
Table I: Best fit values of the standard three neutrino oscillation parameters

\begin{tabular}{cc}
\hline \hline Parameter & Best fit values \\
\hline$\theta_{12}$ & $34.4^{\circ}$ \\
$\theta_{13}$ & $8.50^{\circ}$ \\
$\theta_{23}$ & $45.0^{\circ}$ \\
$\Delta m_{21}{ }^{2}$ & $8 \times 10^{-5} \mathrm{eV}^{2}$ \\
$\Delta m_{31}{ }^{2}$ & $2.5 \times 10^{-3} \mathrm{eV}^{2}$ \\
\hline
\end{tabular}

Table II: Best fit values of sterile mixing angles

\begin{tabular}{cc}
\hline \hline Parameter & Best fit values \\
\hline$\theta_{14}$ & $6.7^{\circ}$ \\
$\theta_{24}$ & $3.3^{\circ}$ \\
$\theta_{34}$ & $6.3^{\circ}$ \\
\hline
\end{tabular}

Table III: Systematics

\begin{tabular}{cc}
\hline \hline Sytematical Errors & values \\
\hline Flux normalization & $2 \%$ \\
Fiducial mass errors for near detector & $0.6 \%$ \\
Fiducial mass errors for far detector & $0.6 \%$ \\
energy calibration error for near detector & $0.5 \%$ \\
energy calibration error for far detector & 0.5 \\
Backgrounds & $10^{-4}$ \\
\hline
\end{tabular}

\section{PROCEDURE}

At the first step we have checked the signature of extrinsic CPT violation at probability level. For this we check the variation of $\triangle P_{\mu \mu}^{C P T}$ as defined by equation (7) with the energy and baseline. An oscillograph shown by Fig.1 illustrates the change in $\triangle P_{\mu \mu}^{C P T}$ with the change in energy and baseline. The normal hierarchy oscillograph quantifies, $\triangle P_{\mu \mu}^{C P T}=+0.0475$ for baselines $4500 \mathrm{~km}$ to $7000 \mathrm{~km}$ in energy range 6 to $11 \mathrm{GeV}, \triangle P_{\mu \mu}^{C P T}=-0.0475$ for baselines $5000 \mathrm{~km}$ to $7000 \mathrm{~km}$ with energy range 20 to $50 \mathrm{GeV}$. The inverted hierarchy oscillograph shown in Fig.2 quantifies $\triangle P_{\mu \mu}^{C P T}=+0.0475$ for baselines $5200 \mathrm{~km}$ to $7000 \mathrm{~km}$ in energy range 18 to $50 \mathrm{GeV}, \Delta P_{\mu \mu}^{C P T}=-0.0475$ for baselines $6000 \mathrm{~km}$ to $7500 \mathrm{~km}$ in energy range 6 to $12 \mathrm{GeV}$. Within three neutrino frame work constraints imposed on $\Delta P_{\mu \mu}^{C P T}$ by MINOS, JHF-Kamioka and K2K are $1 \times 10^{-5}, 1.3 \times$ $10^{-4}$ and $-5.3 \times 10^{-5}$ [13]. For longer baselines like 7500 $\mathrm{km}$ the extrinsic CPT violation is almost negligible for energy $15 \mathrm{GeV}$.

The event rate depends on : the neutrino oscillation probability, type of interaction, source properties and the detector properties. Hence at the second step detector effects are taken into consideration and we have checked the extrinsic CPT violation at rate level by looking at different measurable quantities such as ratio and asymmetry factor. 
(1)The ratio $R$, is the simple ratio between the number of muon neutrinos $N\left(v_{\mu} \rightarrow v_{\mu}\right)$ to the number of muon antineutrinos $N\left(\bar{v}_{\mu} \rightarrow \bar{v}_{\mu}\right)$

$$
R=\frac{N\left(\nu_{\mu} \rightarrow \nu_{\mu}\right)}{N\left(\overline{\nu_{\mu}} \rightarrow \overline{\nu_{\mu}}\right)}
$$

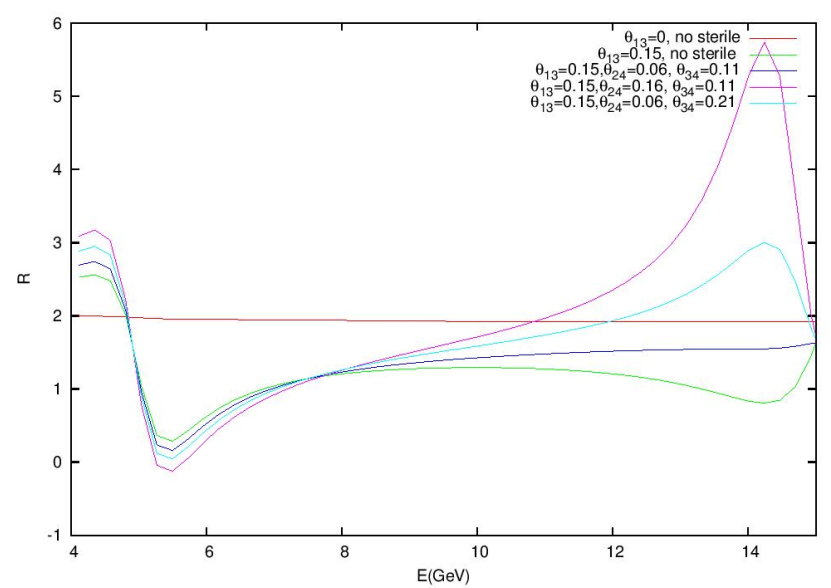

Fig. 3: Variation of R (no. of muon neutrinos / no. of muon antineutrinos) with energy. This observation is made with Liq Argon detector for different set of parameters (i) $\theta_{13}=0$ with no sterile contribution(ii) $\theta_{13}=0.15$ in three neutrino framework (iii)For different values of $\theta_{24}$ and $\theta_{34}$

The variation of ratio $\mathrm{R}$ (number of neutrino events to that of antineutrino events) with respect to energy. This variation is shown in Fig. 3, 4, 7 and 8. Red line shows the crosssection ratio between neutrino and antineutrino. Any deviation from the red line will show the variation of extrinsic CPT violation with energy at baseline $7500 \mathrm{~km}$. To check sterile contribution in the observed deviation, a parameter $\Delta R=(R)_{4 \times 4}-(R)_{3 \times 3}$ is selected. The deviation of $\Delta \mathrm{R}$ from the black line will give a rough measure of extrinsic CPT violation produced by the sterile neutrinos. From Figure 5 and 6 we observe that maximum deviation occurs in the energy range $9-14 \mathrm{GeV}$ and the amount of the deviation enhances when it is observed with Liquid Argon detector in comparison with the MID detector at $7500 \mathrm{~km}$. From Figure 9 and 10 we observe that in energy range 16-20 $\mathrm{GeV}$, CPT violation through sterile neutrino can be observed. Thus providing us the second prominent energy interval of the observance of extrinsic CPT violation arising due to the presence of sterile neutrino.

At the same time these plots suggest that enhanced value of sterile parameters will enhance the extent of extrinsic CPT violation. Hence to deliver the best results out of the above plots we need a well constrained values of sterile parameters.

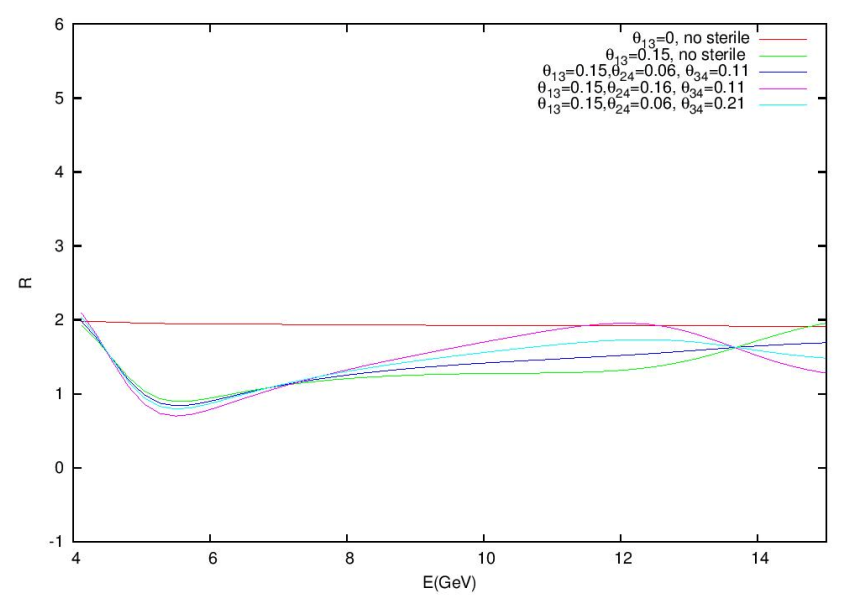

Fig. 4: Variation of R (no. of muon neutrinos / no. of muon antineutrinos) with energy. This observation is made with MID detector for different set of parameters (i) $\theta_{13}=0$ with no sterile contribution(ii) $\theta_{13}=0.15$ in three neutrino framework (iii)For different values of $\theta_{24}$ and $\theta_{34}$

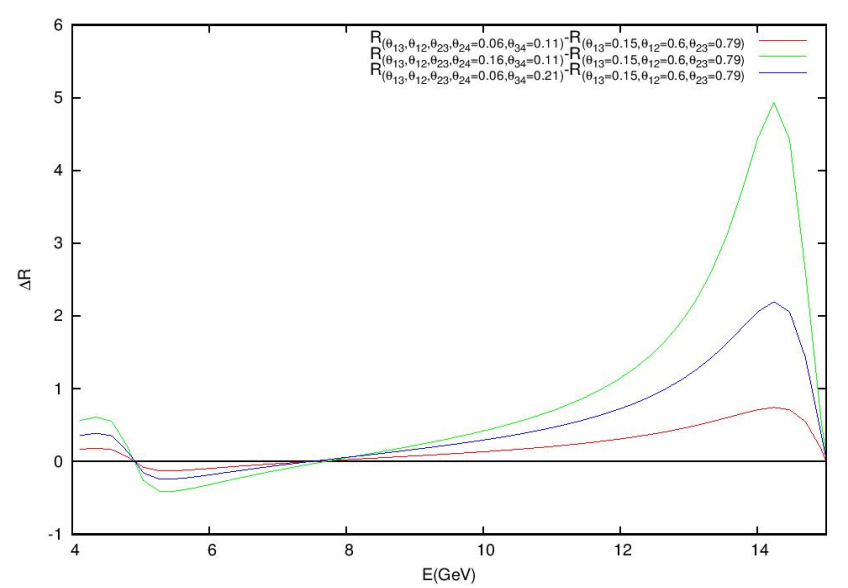

Fig. 5: Variation of $\Delta R$ (no. of muon neutrinos/ no. of muon antineutrinos) with energy. This observation is made with Liq Argon detector for different set of parameters (i) $\theta_{13}=0$ with nosterile contribution (ii) $\theta_{13}=0.15$ in three neutrino framework (iii) For different values of $\theta_{24}$ and $\theta_{34}$

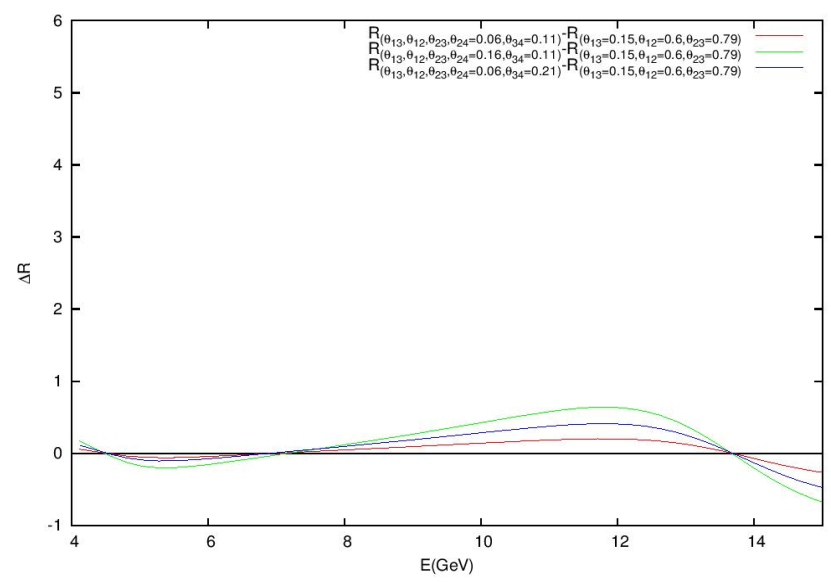

Fig. 6: Variation of $\Delta R$ (no. of muon neutrinos / no. of muon antineutrinos) with energy. This observation is made with MID detector for different set of parameters (i) $\theta_{13}=0$ with no sterile contribution(ii) $\theta_{13}=0.15$ in three neutrino framework (iii)For different values of $\theta_{24}$ and $\theta_{34}$ 


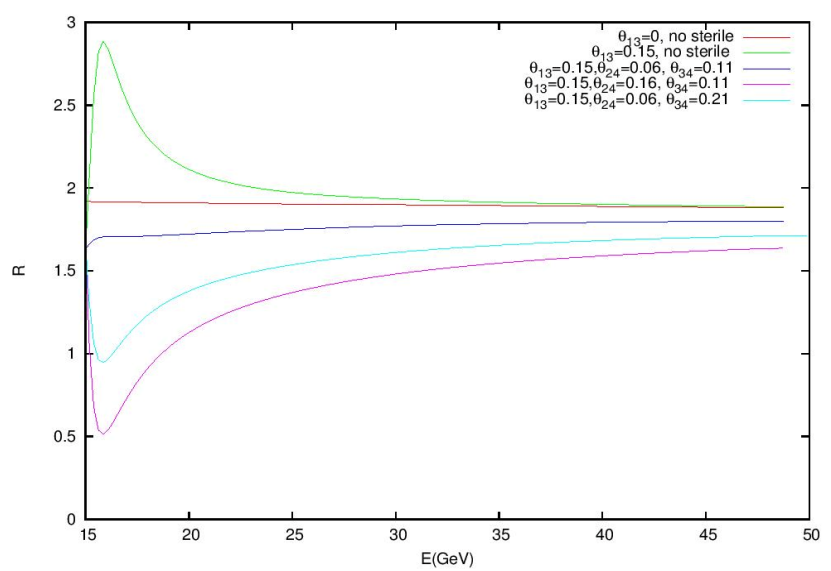

Fig. 7: Variation of R (no. of muon neutrinos / no. of muon antineutrinos) with energy. This observation is made with Liq. Argon detector for different set of parameters (i) $\theta_{13}=0$ with no sterile contribution(ii) $\theta_{13}=0.15$ in three neutrino framework (iii)For different values of $\theta_{24}$ and $\theta_{34}$

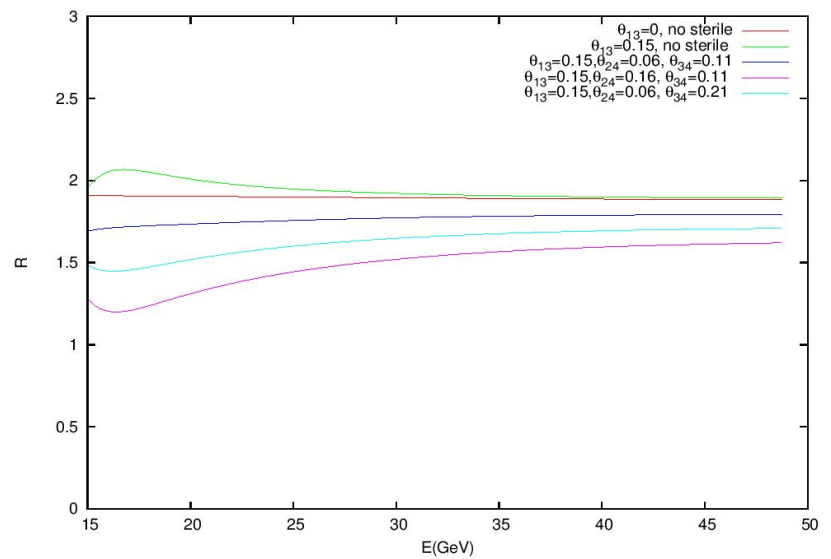

Fig. 8: Variation of R (no. of muon neutrinos / no.. of muon antineutrinos) with energy. This observation is made with MID detector for different set of parameters (i) $\theta_{13}=0$ with no sterile contribution(ii) $\theta_{13}=0.15$ in three neutrino framework (iii) For different values of $\theta_{24}$ and $\theta_{34}$

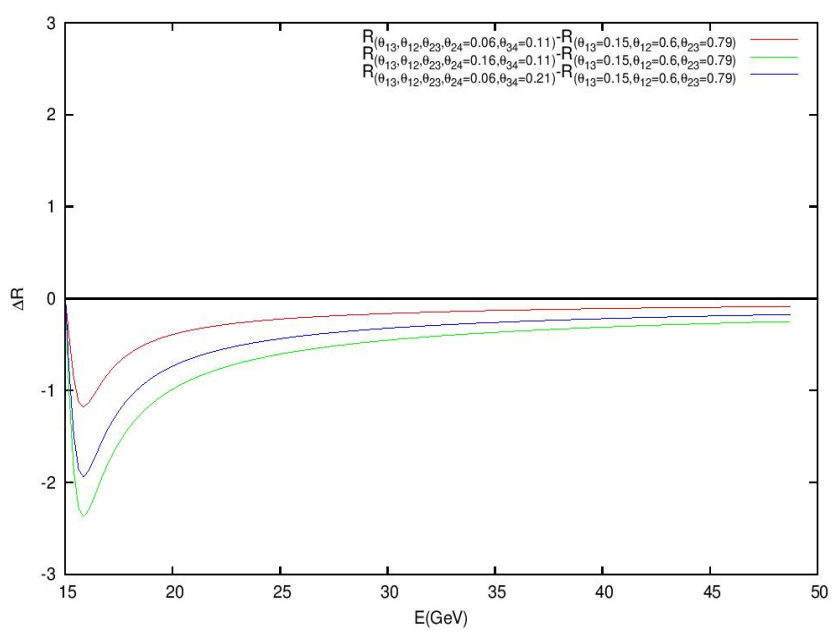

Fig. 9: Variation of $\Delta R$ (no. of muon neutrinos/ no. of muon antineutrinos) with energy. This observation is made with Liq. Argon detector for different set of parameters (i) $\theta_{13}=0$ with nosterile contribution (ii) $\theta_{13}=0.15$ in three neutrino framework (iii) For different values of $\theta_{24}$ and $\theta_{34}$
(2) Asymmetry factor $A_{\mu}$ is the ratio between $N\left(v_{\mu} \rightarrow v_{\mu}\right)$ and $N\left(\bar{v}_{\mu} \rightarrow \bar{v}_{\mu}\right)$ using both far and near detectors.

$A_{\mu}(E) \equiv \frac{N\left(\nu_{\mu} \rightarrow \nu_{\mu}\right)^{\text {far }}(E)}{N\left(\nu_{\mu} \rightarrow \nu_{\mu}\right)^{\text {near }}(E)}-\frac{N\left(\overline{\nu_{\mu}} \rightarrow \overline{\nu_{\mu}}\right)^{\text {far }}(E)}{N\left(\overline{\nu_{\mu}} \rightarrow \overline{\nu_{\mu}}\right)^{\text {near }}(E)}$

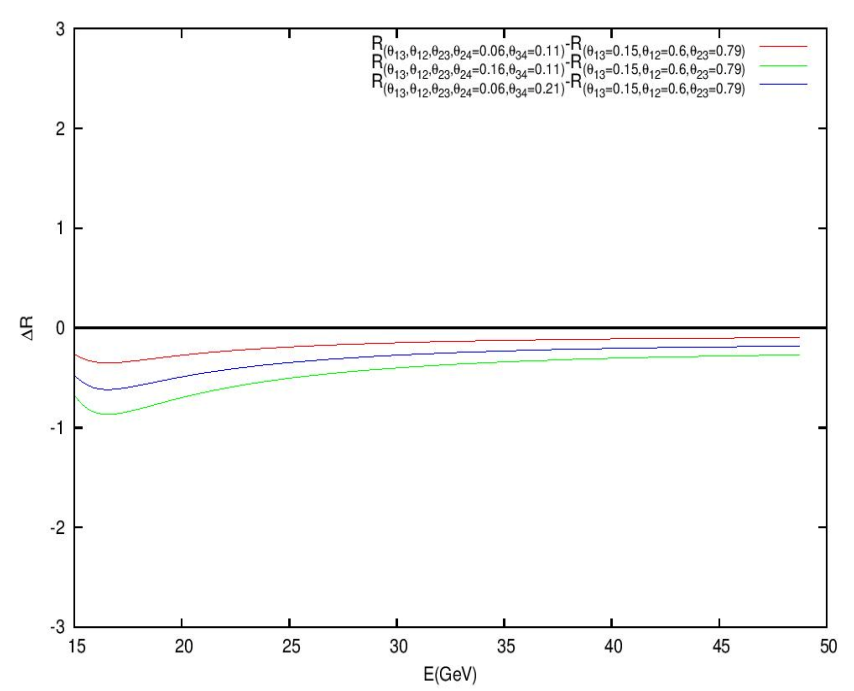

Fig. 10: Variation of $\Delta R$ (no. of muon neutrinos/ no. of muon antineutrinos) with energy. This observation is made with MID detector for different set of parameters (i) $\theta_{13}=0$ with nosterile contribution (ii) $\theta_{13}=0.15$ in three neutrino framework (iii) For different values of $\theta_{24}$ and $\theta_{34}$

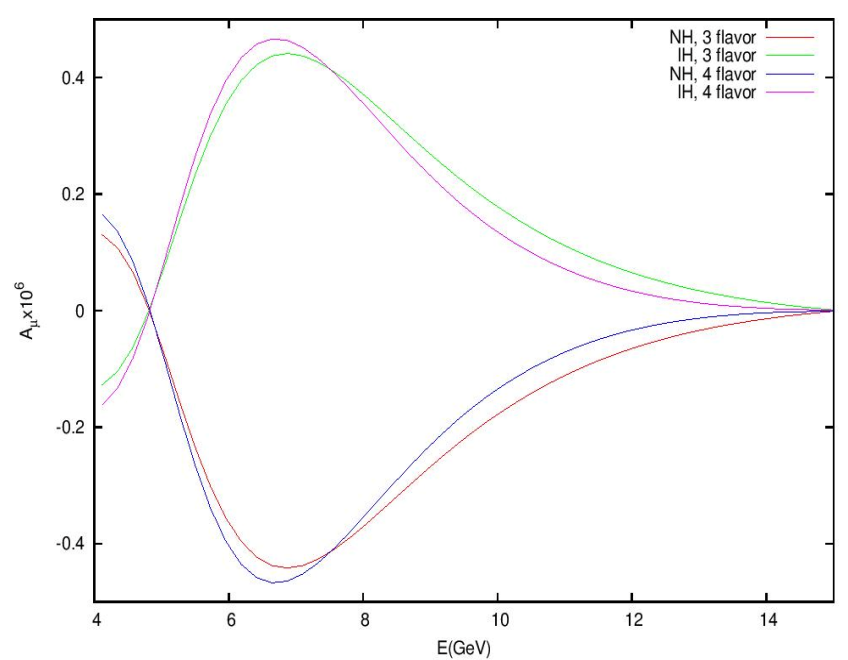

Fig. 11: Variation in asymmetry factor as a function of energy, observed with Liq. Argon detector. Both near and far detectors are used for the observation. Near detector is placed at $20 \mathrm{~m}$ and far detector is placed at $7500 \mathrm{~km}$

This asymmetry factor is roughly equal to $A_{\mu} \approx P_{\mu \mu}-P_{\overline{\mu \mu}}$. The variation of asymmetry with energy is shown by figures $11,12,13$ and 14 . Looking at figure 11 and 12 we notice that asymmetry is large in energy range $6-8 \mathrm{GeV}$ and similarly from figure 13 and 14 we notice that beyond $15 \mathrm{GeV}$ asymmetry is low in general. But in the present work we are looking for the sterile contribution to the total $\mathrm{CPT}$ violation 
observed. Keeping this in mind the energy region of our interest is the region where separation between $A_{\mu(3 \times 3)}$ and $A_{\mu(4 \times 4)}$ for both the hierarchies is high. The separation between $A_{\mu(3 \times 3)}$ and $A_{\mu(4 \times 4)}$ will reflect the contribution of sterile neutrino to the total extrinsic CPT violation, more the separation more will be the contribution from sterile neutrino . Again looking at Figures 11, 12, 13 and 14 we found that the separation between $\left(A_{\mu}\right)_{4 \times 4}$ and $\left(A_{\mu}\right)_{3 \times 3}$ for both hierarchies is prominent in the energy interval 9-13 $\mathrm{GeV}$ whereas at $15 \mathrm{GeV}$ this separation is very less nearing to zero. At the same time looking at asymmetry plot shown by Figure 12 which is generated by using MID detector as particle detector, we perceive that for normal hierarchy(NH) this separation is very less. Therefore if $\mathrm{NH}$ is true hierarchy MID detector will not be a suitable detector to probe contribution of sterile neutrino in extrinsic CPT violation with neutrino factory at long baselines. The energy range 9 GeV-10 GeV is sensitive for catching extrinsic CPT violation generated by sterile neutrinos and the sensitivity at these energies can also be observed from probability oscillographs. Hence from this we stipulate that lower energies (till $14 \mathrm{GeV}$ ) are not suitable for examining the signatures of pure CPT violation in presence of sterile neutrino since in this energy range CPT violation contribution arising from matter effects is high.

As sterile parameters still needs a stringent constraints from all available and coming neutrino experiments. In an endeavour to get realistic estimation of extrinsic CPT violation the product of sterile angles $\left(\theta_{24} \cdot \theta_{34}\right)$ are constrained using neutrino factory at $90 \%$ C.L. To achieve better fits we have marginalized $\Delta m_{31}{ }^{2}$ and $\delta_{C P}$ in $3 \sigma$ range. Contour plot shown in fig. 15 imposes constrain on the product of sterile angles $\theta_{24}$ and $\theta_{34}$ as $\theta_{24} \cdot \theta_{34} \gtrsim 0.006$ when observed with Liquid Argon detector and $\theta_{24} \cdot \theta_{34} \geq$ 0.007 with MID detector.

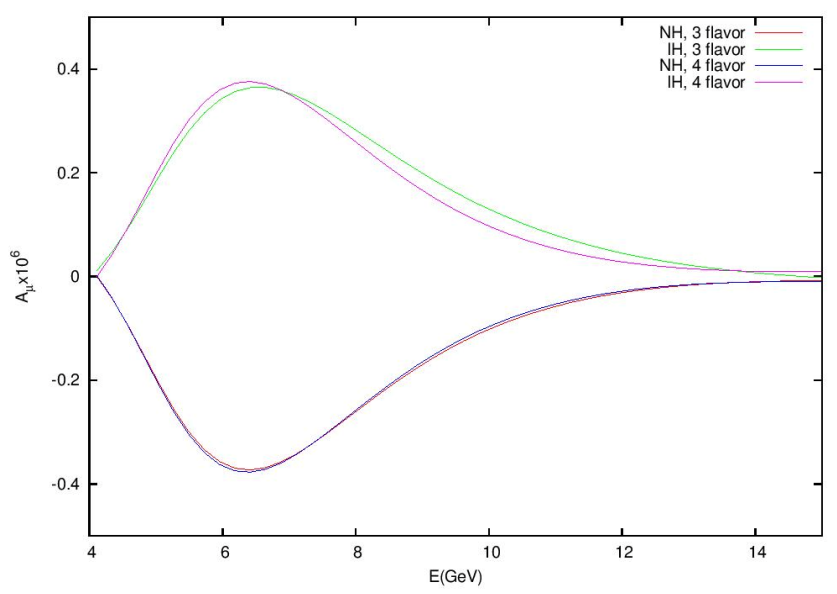

Fig. 12: Variation in asymmetry factor as a function of energy, observed with MID detector. Both near and far detectors are used for the observation. Near detector is placed at $20 \mathrm{~m}$ and far detector is placed at $7500 \mathrm{~km}$

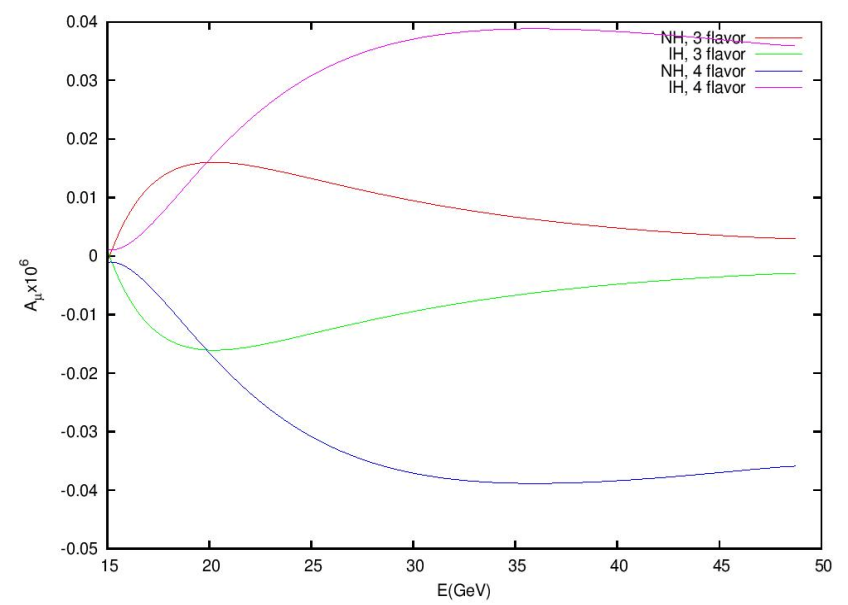

Fig. 13: Variation in asymmetry factor as a function of energy, observed with Liq. Argon detector. Both near and far detectors are used for the observation. Near detector is placed at $20 \mathrm{~m}$ and far detector is placed at $7500 \mathrm{~km}$

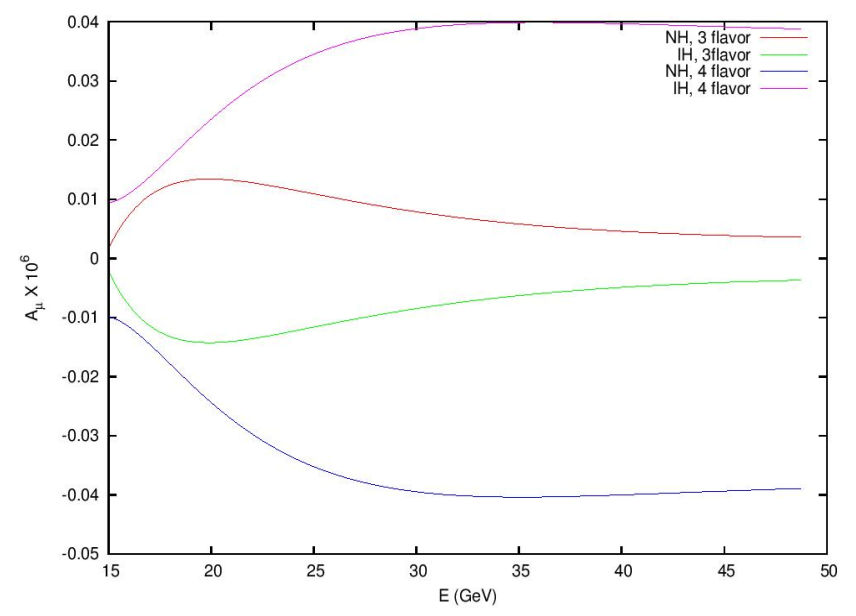

Fig. 14: Variation in asymmetry factor as a function of energy, observed with MID detector. Both near and far detectors are used for the observation. Near detector is placed at $20 \mathrm{~m}$ and far detector is placed at $7500 \mathrm{~km}$

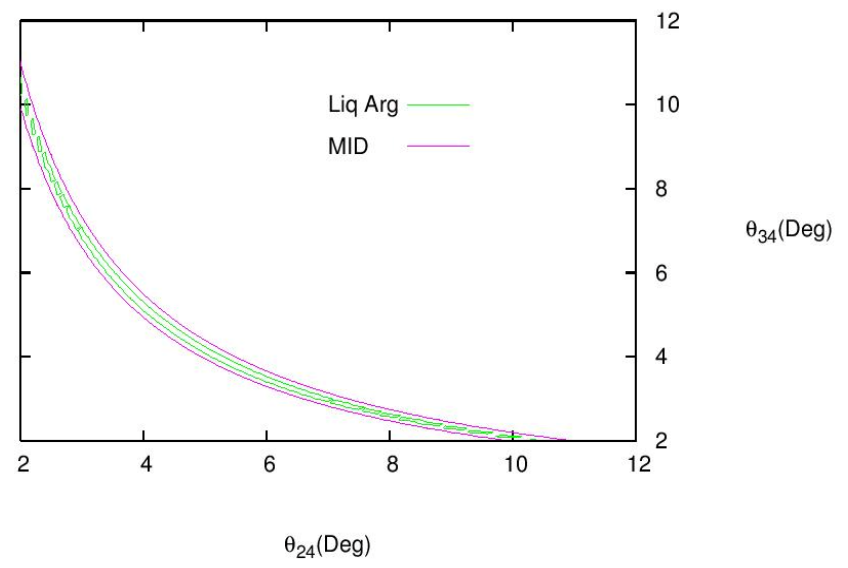

Fig. 15: Contours in the $\theta_{24}-\theta_{34}$ plane at the $90 \%$ C.L. The two curves corresponds to Liq. Arg and MID detector. Here only far detector is used which is placed at magic baseline $7500 \mathrm{~km}$ 


\section{RESULT AND DISCUSSION}

We have checked the potential of neutrino factory to quantify the extrinsic CPT violation in four flavor neutrino framework. In this work initial check for the presence of CPT violation is observed at the probability level. In order to filter out extrinsic CPT violation arising due to the presence of sterile neutrino from those which arises from the normal three neutrino frame work, we select the CPT probability difference as $\Delta P_{\alpha \beta}^{C P T}=$ $\left(P_{\alpha \beta}-P_{\bar{\beta} \bar{\alpha}}\right)_{4 v}-\left(P_{\alpha \beta}-P_{\bar{\beta} \bar{\alpha}}\right)_{3 v}$. Here PREM matter density profile is used for matter through which the neutrinos are travelling. From the above work we infer that in presence of sterile neutrino, baseline till $2000 \mathrm{~km}$ is safe to carry out experiments looking for pure CPT violation(the intrinsic or pure CPT violation arises due to the violation of CPT invariance theorem) as for baselines below $2000 \mathrm{~km}$ extrinsic CPT violation arising due to the presence of sterile neutrino is negligible. In order to extract sterile contribution from R Vs E and $A_{\mu} \mathrm{Vs}$ E plot we look at parameters $\Delta R$ and $\left(A_{\mu}\right)_{4 \times 4}-\left(A_{\mu}\right)_{3 \times 3}$ for both the hierarchies. These plots corresponds to $7500 \mathrm{~km}$ baseline. From these plots we surmise that with increasing energy extrinsic CPT violating effects arising due to sterile neutrino decreases significantly, especially at $15 \mathrm{GeV}$ these effects are almost negligible. Hence $15 \mathrm{GeV}$ or higher energies are suitable to probe pure CPT violating effects since at these energies the contribution of sterile neutrino to total extrinsic CPT violation becomes very less. At the same time we conclude that extrinsic CPT violation increases with the increase in the value of sterile angles. Hence in the presence of sterile neutrino the correct estimation of extrinsic CPT violation can only be achieved if we are able to impose proper constraints on sterile parameters. For observing genuine sterile contribution to CPT violation the product of sterile angles which appear in the selected $\left(v_{\mu} \rightarrow v_{\mu}\right)$ channel are constrained as $\theta_{24} \cdot \theta_{34} \geq 0.006$ with Liquid argon detector and $\theta_{24} \cdot \theta_{34} \geq$ 0.007 with magnetized iron detector at $90 \%$ confidence level. These results advocates that with Liquid argon detector we can impose better constraint on sterile parameters which in turn will impose better constrain on the extent of extrinsic CPT violation.

\section{ACKNOWLEDGMENT}

One of the authors Sujata Diwakar is thankful to University Grant Commission, India for giving financial support under the Rajiv Gandhi National Fellowship scheme.

\section{REFERENCES}

[1] Q.R. Ahmad et al. Phys. Rev. Lett. 89(2002)011302

[2] S.N. Ahmed et al. submitted to Phys. Rev. Lett.(2003), nuclex/0309004

[3] Super-Kamiokande Collaboration, Y. Fukuda et al., Phys. Rev. Lett. 81, 1562 (1998); 81, 4279 (1998); S. Fukuda etal., Phys.Lett. B 539, 179 (2002)

[4] C. Athanassopoulos et al. [LSND collaboration], Phys Rev. Lett. 77 (1996) 3082 [arXiv : nucl-ex /9605003].
[5] C. Athanassopoulos et al. [LSND collaboration], Phys. Rev. Lett. 75 (1995) 2650.

[6] C. Athanassopoulos et al. [LSND collaboration], Phys Rev. Lett. 81 (1998) 1774 [arXiv:nucl-ex/9709006].

[7] C. Athanassopoulos et al. [LSND collaboration], Phys. Rev. C 58 (1998) 2489.

[8] A.Aguilar et al. [LSND Collaboration], Phys. Rev. D 64 (2001) 112007[arXiv:hep-ex/0104049].

[9] C. Athanassopoulos et al. [LSND collaboration], Phys. Rev Lett 77 (1996) 3082, [arXiv nucl-ex /9605003].

[10] [ALEPH, DELPHI, L3, OPAL, and SLD collaborations],Phys. Rep.427, 257 (2006)

[11] W.M Yao et al. [Particle data group], J. Phys. G 33, 1 (2006).

[12] K. Nakeamura et al., Review of Particle Physics, J. Phys.G37 (2010) 075021 [SPIRES].

[13] Magnus Jacobson and Tommy Ohlsson, arXiv hep$\mathrm{ph} / 0305064 \mathrm{v} 3$

[14] Animesh Chatterjee, Raj Gandhi, Jyotsna Singh, arXiv: 1402.6265v1[hep-ph](2014)

[15] A. Samanta, Phys. Lett. B 693, 296 (2010) arXiv:1005.4851[hep-ph]

[16] V. D. Barger, S. Pakvasa, T. J. Weiler and K. Whisnant, Phys. Rev. Lett. 85, 5055(2000) [hep-ph/0005197].

[17] Anindya Datta, Raj Gandhi, Poonam Mehta and S Uma Sankar arXiv:hep-ph/0312027v2(2004)

[18] M. C. Gonzalez-Garcia, M. Maltoni, T. Schwetz, arXiv:hepph/1409.543v1.

[19] Davide Meloni, Jian Tang and Walter Winter, arXiv:hepph/1007.2419v2.

[20] Steve Geer, hep-ph/0008155v1

[21] International Design study of the neutrino factory, http://www.ids-nf.org.

[22] P. Huber, M. Lindner, M. Rolinec and W. Winter, Phys. Rev. D 74, 073003 (2006), hep-ph/0606119.

[23] P. Huber, M. Lindner and W. Winter, Nucl. Phys. B 6453(2002), hep-ph/0204352.

[24] E. Ables et al.(MINOS) FERMILAB-PROPOSAL-P875

[25] C. H. Albright et al. [Neutrino Factory / Muon Collider Collaboration], arXiv:Physics/0411123.

[26] Jian Tang, Walter Winter, arXiv:0903.3039v2.

[27] P. Huber, M. Lindner and W. Winter, Comput. Phys. Commun. 167,195 (2005), http://www.mpihd.mpg.de/Lin/globes/; hep-ph/0407333.

[28] P. Huber, J. Kopp, M. Lindner, M. Rolinec and W.Winter Comput. Phys. Commun. 177, 432 (2007), hepph/ 07071187.

[29] R. J. Geller and T. Hara, Phys. Rev. Lett. 49, 98 (2001),hep-ph/0111342.

[30] T. Ohlsson and W. Winter, Phys. Rev. D 68, 073007

(2003), hep-ph/0307178. 